\title{
Preferencias relacionales en la adolescencia según el género
}

\section{Relationship Preferences in Adolescence According to Gender}

Ángeles Rebollo-Catalán (*) rebollo@us.es

Estrella Ruiz-Pinto (*) epinto@us.es

Rafael García-Pérez (*) rafaelgarcia@us.es

(*) Universidad de Sevilla

(Recibido: 12 de diciembre de 2014; Aceptado para su publicación: 5 de noviembre de 2015)

Cómo citar: Rebollo-Catalán, A., Ruiz-Pinto, E. y García-Pérez, R. (2017). Preferencias relacionales en la adolescencia según el género. Revista Electrónica de Investigación Educativa, 19(1), 58-72. Recuperado de https://doi.org/10.24320/redie.2017.19.1.1022

\section{Resumen}

El artículo presenta los resultados de un estudio sobre las relaciones de género en la adolescencia. El objetivo fundamental es conocer la predisposición del alumnado a establecer relaciones entre iguales según la naturaleza de la actividad y analizar la influencia de expectativas sociales de género en sus elecciones. Se aplica una escala, diseñada ad hoc, para medir las preferencias relacionales. Participaron 722 estudiantes de Educación Secundaria de 12 a 17 años. Los resultados muestran que los chicos son más elegidos para jugar videojuegos y practicar deporte, y las chicas lo son para compartir secretos y prestarse ropa. También se observa una predisposición a elecciones mutuas entre adolescentes del mismo sexo en actividades escolares y extraescolares. Los resultados obtenidos muestran coincidencias con investigaciones realizadas en contextos diferentes, lo que demuestra la influencia de estereotipos de género en las relaciones entre iguales durante la adolescencia.

Palabras clave: Adolescencia, relaciones entre iguales, diferencias de género, estereotipos de género.

\section{Abstract}

This paper offers the results of a study of gender relations in adolescence. The main aim is to determine students' propensity to establish relationships with peers based on the nature of the activity, and analyze the influence of social expectations of gender in their choices. An ad hoc scale was designed and used to measure relationship preferences. The study sample was made up of 722 secondary education students aged between 12 and 17 . The results show that boys are favored to play videogames and in sports activities, and girls are preferred to share secrets and swap clothes. The study also observes a tendency towards reciprocal choices between students of the same sex in school and extracurricular activities. The results obtained coincide with research in different contexts, which shows the impact of gender stereotypes on peer relationships in adolescence. 
Keywords: Adolescence, peer relations, dender differences, gender stereotypes.

\section{Introducción}

La adolescencia es un período crucial para el establecimiento de relaciones de género (Bascón, Arias y De la Mata, 2013; Garaigordobil, 2012), ya que en esta etapa se produce una consolidación de las habilidades sociales, la expansión de relaciones entre iguales, el fraguado de nuevas redes sociales y la constitución de grupos y parejas sentimentales.

Feiring (1999) observó que las personas que referían mayor número de amistades del sexo contrario en su infancia disponían de mejores capacidades y destrezas para las relaciones de pareja, al haberse socializado en la confianza, la reciprocidad y el apoyo mutuo durante sus interacciones y relaciones amistosas. En este sentido, Navarro-Pertusa (2004) señala que el establecimiento de relaciones amistosas entre personas de distinto sexo en la infancia y adolescencia supone un aprendizaje de habilidades relacionales tales como la negociación y la resolución de conflictos.

Algunos estudios (Benenson y Christakos, 2003; Parker, Low, Walker y Gamm, 2005; Rose, Carlson y Waller, 2007) han destacado diferencias significativas entre chicos y chicas en la configuración de las relaciones amistosas que se empiezan a establecer en la infancia y que continúan durante la adolescencia, las cuales surgen antes en el tiempo en las chicas que en los chicos. En su revisión sobre el estado de la investigación en género y adolescencia, Perry y Pauletti (2011) encuentran que las relaciones de amistad entre las chicas están basadas en el cuidado, son de gran intimidad y confianza, se caracterizan por compartir problemas y sentimientos, mientras que las relaciones amistosas entre chicos están marcadas por la competición amigable, las actividades de riesgo, los esfuerzos de control directo y la inhibición de los sentimientos e intimidad.

Algunos trabajos han mostrado carencias en el ámbito de las relaciones entre iguales durante la adolescencia (De Lemus, Castillo, Moya, Padilla y Ryan, 2008; Garaigordobil y Aliri, 2013; Leaper y Brown, 2008). En su estudio sobre actitudes del alumnado hacia la igualdad de género, GarcíaPérez et al. (2010) muestran que el alumnado obtiene puntuaciones más bajas en el plano relacional, siendo el ámbito donde se acumulan las mayores diferencias entre chicos y chicas; esto es, las puntuaciones de los chicos se sitúan en niveles próximos al sexismo y, especialmente, en indicadores relativos a la justificación de la violencia hacia las mujeres. Herrera, Expósito y Moya (2012) también encuentran que las actitudes y comportamientos de chicos adolescentes y jóvenes se acercan a posiciones de sexismo hostil en las relaciones de pareja cuando pierden el poder y el control y las mujeres no aceptan sus decisiones.

En este sentido, el estudio de Martínez-Benlloch, Bonilla-Campos, Gómez-Sánchez y Bayot (2008) revela diferencias entre discursos y prácticas de chicos y chicas adolescentes, encontrando que conviven, por un lado, la aceptación de la igualdad formal junto con manifestaciones de sexismo benevolente en el plano real de las relaciones entre hombres y mujeres, legitimando en la práctica la subordinación de las mujeres y abogando por la pervivencia del rol femenino en el ámbito de las relaciones afectivo-sexuales. De la Peña, Ramos, Luzón y Recio (2011) muestran el elevado porcentaje de adolescentes de Andalucía que ven la realidad desde una óptica sexista, observando un efecto evolutivo visible de disminución del sexismo en grupos de mayor edad, lo que atribuyen a la influencia de las primeras relaciones amorosas en la adolescencia y el cambio que ello supone en la visión e interacción con el otro sexo. De la Peña et al. (2011) también encuentran que el sexismo está más interiorizado en lo que se refiere a roles, es decir, a las tareas, funciones, actividades y responsabilidades propios de uno y otro sexo, siendo más fuerte su presencia en los chicos que en las chicas.

En cuanto a las relaciones adolescentes, Muñoz-Tinoco, Jiménez-Lagares y Moreno (2008) encuentran diferencias de género, observando que mientras las chicas obtienen puntuaciones más altas en sociabilidad (ayudar, preocuparse, promover diálogos y acuerdos), los chicos lo hacen en agresividad física y relacional (meter en líos a otras personas y manipular a los demás 
para conseguir sus objetivos). Ruiz-Pinto, García-Pérez y Rebollo-Catalán (2013) encuentran que estudiantes adolescentes eligen más a las chicas para realizar tareas basadas en el cuidado, mientras prefieren chicos para actividades de competición; mostrando además que las redes relacionales para el cuidado son más frágiles y dispersas, mientras que las que implican competir son más fuertes y cohesionadas.

Otros estudios (Mendoza, Batista-Foguet y Rubio, 2006; Monjas, Sureda y García-Bacete, 2008; Soler, 2009) han mostrado la influencia de estereotipos de género en las actividades e interacciones durante la adolescencia, encontrando incluso una fuerte resistencia al establecimiento de relaciones entre iguales basadas en principios no discriminatorios. Así, Soler (2009) muestra estos comportamientos estereotipados en las clases de Educación Física en el contexto escolar, en el que los chicos optan por compartir la actividad de jugar al fútbol con otros chicos varones y cuando participan chicas no las tratan con la misma consideración. Mendoza et al. (2006) también observa diferencias de género en la práctica de las tareas domésticas cotidianas en adolescentes, mostrando la mayor asunción de responsabilidades por las chicas. Los motivos por los que niños y niñas se aceptan y se rechazan en el grupo de iguales, es decir, por qué establecen -o no- relaciones con sus iguales ha sido investigado (García-Bacete, Sureda y Monjas, 2010; Monjas et al., 2008), puesto que obtener esta información es importante para averiguar la cantidad y el tipo de carga sociocultural (estereotipos, valores, creencias, actitudes, comportamientos, etc.) que influye en el establecimiento de las relaciones.

En el plano relacional durante la adolescencia, Inglés et al. (2008) también encuentra diferencias de género, observando que los chicos se manifiestan, mayoritariamente, mediante conductas agresivas y que las chicas destacan por conductas prosociales. Asimismo, en su estudio, Garaigordobil (2012) comprueba que las chicas obtienen puntuaciones significativamente superiores que los chicos en la resolución de conflictos de manera cooperativa, mientras que los chicos adolescentes destacan en la resolución de conflictos de forma agresiva. En su estudio, Noakes y Rinaldi (2006) también encuentran diferencias de género en los conflictos durante la adolescencia, hallando que las chicas tienen más conflictos de índole relacional y usan estrategias para mitigarlos, mientras que los chicos tienen conflictos relacionados con el estatus y la dominación y usan estrategias de confrontación. Bascón, Saavedra y Arias (2013) documentan la presencia de conflictos vinculados a estereotipos de género en la adolescencia, referidos a la asunción de responsabilidad en las chicas y la exención de culpabilidad en los chicos, la supremacía y control de los hombres sobre las mujeres, y la presencia de los mitos del amor romántico en las relaciones de pareja ligados a la falta de autonomía de las mujeres.

Estos trabajos muestran la adolescencia como una etapa importante para el desarrollo de habilidades y competencias relacionales, así como para la interiorización de creencias y valores compatibles con la igualdad de género, revelando la influencia social en las actividades que se realizan y las interacciones que se establecen durante la adolescencia. Las investigaciones revisadas ponen de manifiesto la enorme tarea educativa que aún queda por hacer en este terreno. Creemos que el presente estudio cubre una laguna en varios sentidos: aporta conocimiento sobre el establecimiento de relaciones entre adolescentes, y descubre si sus preferencias relacionales muestran patrones de género diferenciales que puedan atribuirse a estereotipos de género.

Muchas de las investigaciones previas sobre relaciones de género en la adolescencia se remontan a más de una década, cambiando en los últimos años las prácticas sociales de comunicación e interacción con la irrupción de nuevos medios sociales, que inciden de manera especial en la adolescencia. Por otro lado, las investigaciones más recientes se han centrado en medir el sexismo y la violencia de género, pero no tanto en constatar los motivos y actividades para los cuales los adolescentes establecen relaciones mutuas en el seno de la escuela. Nuestro estudio muestra hasta qué punto la escuela actual es verdaderamente coeducativa al aportar información sobre la naturaleza de las actividades que los adolescentes comparten y en las que colaboran. 


\section{1 Socialización del género}

Como se señala en el apartado anterior, no sólo las creencias y actitudes sino los comportamientos y relaciones de adolescentes y jóvenes están condicionadas por lo que es socialmente deseable. Diversos trabajos han analizado la socialización diferencial de género y las atribuciones del rol de género.

Bosch, Ferrer y Alzamora (2006) afirman que esta socialización se caracteriza por asignar actividades y roles diferenciados a hombres y mujeres, de manera que se educa a chicos y chicas para que se comporten de formas diferentes y en ámbitos de actividad distintos, atribuyendo además un valor social desigual a lo masculino y lo femenino. Este aprendizaje de la masculinidad y la feminidad como modelos universales, dicotómicos y opuestos entre sí se hace por influencia social (Barberá, 2006; Freixas, 2001; Rebollo-Catalán, 2006; Rodríguez-Mosquera, 2011; Sau, 2000). Lomas (2007) añade que hombres y mujeres somos diferentes no sólo porque tengamos un sexo distinto, sino porque aprendemos a ser hombres y a ser mujeres de maneras diferentes como consecuencia del influjo de mediaciones subjetivas y culturales (el origen sexual, el estatus económico y social, la familia, la instrucción escolar, el lenguaje, los estilos de vida, las creencias, etc.) que influyen en la construcción de la identidad. Según Simón (2010), las atribuciones de los roles de género que hombres y mujeres hemos aprendido y repetido desde hace siglos, ha llevado a considerar el rol de reproducción y cuidado como una función social de las mujeres, mientras que el rol de producción y mando es un ámbito asociado con los hombres.

Algunos estudios han documentado esta influencia social respecto a la masculinidad en los comportamientos y relaciones de los hombres (Bonino, 2000; Lomas, 2007; Lorente, 2009; Oransky y Marecek, 2009). En su estudio, Sabuco, Sala, Santana y Rebollo-Catalán (2013) evidencian que los chicos adolescentes asocian la masculinidad al control, la fuerza, la seguridad y la independencia, afianzando su identidad masculina por oposición a los significados de feminidad y de las conductas propias de las chicas. Por su parte, Olavarría (2004) y Callirgos (2003) muestran que en el proceso de hacerse hombre socialmente el adolescente emplea ciertos rituales y prácticas para afirmar su masculinidad principalmente ante sus iguales, tales como las peleas con otros chicos, la utilización de un lenguaje ingeniosamente agresivo, el uso de apodos -en su mayoría insultantes-, la búsqueda de destrezas en el deporte, el uso de símbolos de poder como pueden ser la vestimenta, el estilo de peinado, el habla, la astucia y la propia fortaleza física. En este sentido, Lomas (2007) señala la importancia de la "tribu masculina" en la configuración de la identidad masculina. Subirats y Tomé (2007) también documentan cómo los estereotipos de género influyen en la educación de los varones apoyándose en la competición, en la exigencia de sobresalir y ser mejor que los demás, transmitiéndoles valores como la fuerza, la imposición y la dureza a través de actividades como la práctica de determinadas modalidades deportivas, incitándoles incluso a veces al enfrentamiento o la agresividad como forma de resolución de conflictos.

Por su parte, algunas investigadoras feministas (Bosch, Ferrer, Ferreiro y Navarro, 2013; Coria, 2005; Freixas, 2001; Lagarde, 2000; Simón, 2008) aportan evidencias de cómo las atribuciones sociales respecto a la feminidad han condicionado la vida de las mujeres, denunciado la presencia de un sistema social que limita sus posibilidades de desarrollo. Estas creencias y desigualdades sociales inciden en las expectativas, el uso del tiempo, las ocupaciones, etc. El Instituto Nacional de Estadística (2013) efectuó un estudio que aporta datos esclarecedores a este respecto: el 91.9\% de las mujeres dedica 4:29 horas al día a las tareas domésticas y ocuparse del cuidado de menores de edad, personas mayores y personas dependientes; frente al $74.7 \%$ de los hombres, que dedica una media de 2:32 horas al día al hogar y la familia. Además, algunos estudios (Chaves, 2002; Mañeru, 2007) muestran que las mujeres apoyan en mayor medida sus relaciones en la comunicación, el apoyo mutuo y el reconocimiento recíproco.

Estos estudios han demostrado la influencia social de estereotipos de género que proponen una lógica de cuidado y afecto en la construcción social de la feminidad y una lógica de dominio, competición y control en la construcción social de la masculinidad. Con este trabajo, queremos 
saber hasta qué punto éstos influyen en la predisposición del alumnado adolescente de Educación Secundaria en sus actividades y relaciones entre iguales.

En definitiva, el propósito general de este trabajo es estudiar las relaciones de género en la adolescencia, considerando, para ello, como objetivo: conocer la predisposición del alumnado adolescente a establecer relaciones entre iguales según la naturaleza de la actividad y analizar la influencia de estereotipos de género en el establecimiento de sus relaciones. Tomando como base la argumentación teórica presentada, se hipotetiza que en términos generales los adolescentes prefieren elegir a chicas para actividades basadas en el cuidado y a chicos para actividades basadas en la competición. De forma complementaria, buscamos comprobar si existen diferencias en función del género y la edad en las elecciones.

\section{Método}

Este trabajo surge en el marco de un proyecto más amplio, cuyo propósito ha sido realizar un diagnóstico de los centros educativos andaluces en materia de igualdad, del cual deriva el propósito de analizar las relaciones de género en la adolescencia y, específicamente la predisposición del alumnado a elegir chicos o chicas en función de la naturaleza de las actividades con el fin de conocer la posible incidencia de las atribuciones sociales de los roles de género.

Participaron en el estudio 722 adolescentes de 12 a 17 años (72 estudiantes no anotaron su edad) que cursan estudios de Educación Secundaria Obligatoria (ESO) en 10 centros públicos de Sevilla (Andalucía, España) durante el curso académico 2009-2010, de los cuales 305 cursan 1o. de ESO, 188 20. de ESO, 94 3o. de ESO y 135 4o. de ESO. Participaron 355 chicos y 355 chicas (12 estudiantes no especificaron sexo). El tamaño ( $n=722$ ) permite trabajar con un error muestral de $3.7 \%$, para un nivel de confianza del $95.5 \%$ con la máxima varianza proporcional $p=q=50 \%$, en una población de carácter infinito (>100,000 casos). La muestra se ha seleccionado siguiendo un procedimiento de muestreo aleatorio estratificado y por conglomerados, considerando tres estratos en la población en función de la zona geográfica (zona urbana, zona peri-urbana y zona rural) del centro educativo y tomando como conglomerados los centros educativos que imparten sus enseñanzas en cada zona.

Se aplicó una escala diseñada ad hoc para medir las preferencias relacionales de género en la adolescencia, la cual está estructurada en cuatro preguntas: 1) para qué elegirías a una chica, 2) para qué elegirías a un chico, 3) para qué no elegirías a una chica y 4) para que no elegirías a un chico. Cada pregunta presenta un rango de respuesta de diez opciones que muestran distintas actividades: tener una relación de pareja, estudiar juntos/as, compartir un secreto, sentarnos juntos/as en clase, prestarnos la ropa, ordenar y limpiar, practicar un deporte juntos/as, jugar a un videojuego, ser delegado/a de la clase, e ir de acampada. La pauta de respuesta en cada pregunta permite elegir tantas opciones como el alumnado desee.

Рara el diseño de este instrumento se contemplaron actividades propias para su edad en el ámbito escolar, considerando actividades que supongan también un contexto relacional marcado por atribuciones sociales de género; con la intención de explorar las preferencias relacionales de género en función de la naturaleza de las actividades. Para asegurar la validez de contenido del instrumento se elaboró una lista de control a partir de la revisión de investigaciones previas sobre actividades de interacción entre iguales durante la adolescencia (Oliva et al., 2007; Salvador, 2009; Simón, 2010; Subirats y Tomé, 2007), la cual fue sometida al juicio de personas expertas en género y adolescencia del ámbito de la psicología y la educación, lo que permitió perfilar el contenido y redacción de la escala.

Se concertó con los equipos directivos de los centros educativos participantes en el estudio la aplicación de la "Escala de Preferencias Relacionales de Género" en el horario semanal dedicado a tutorías en cada grupo-clase. El tiempo de realización de la prueba fue de 30 minutos en cada grupo-clase. La escala se aplicó de forma colectiva a cada grupo en horario lectivo contando con 
la colaboración del profesorado-tutor. El alumnado fue informado del carácter voluntario y anónimo de su participación en el estudio y de los objetivos del mismo, y fue tratado respetando las normas éticas al uso en la realización de una investigación con menores.

Una vez introducidos los datos en el paquete estadístico SPSS (versión 18 para Windows), se realizó un análisis exploratorio de las variables para conocer su distribución (frecuencias y porcentajes). Además, se aplicó la chi-cuadrada como prueba de contraste para probar las diferencias en función del género y la edad y el coeficiente de contingencia para estudiar la asociación entre variables de escala nominal. Para este trabajo se presentan exclusivamente los resultados de preferencias relacionales de elección y no los de rechazo.

\section{Resultados}

Los resultados muestran que las chicas son elegidas en un alto porcentaje para sentarse juntos (77\%), estudiar juntos (73.3\%) y compartir un secreto (69.3\%), mientras que los chicos lo son para sentarse juntos (70.2\%), practicar deporte juntos (66.6\%), jugar a videojuegos (66.4\%) y estudiar juntos (61.6\%). Estos resultados muestran que chicos y chicas son elegidos en un alto porcentaje para actividades propias del contexto escolar como sentarse juntos o estudiar juntos. Sin embargo, se observa que una de las actividades para las que más se elige a las chicas es para compartir secretos, mientras que las actividades en las que más se elige a los chicos son para jugar videojuegos y practicar deporte. La tabla I resume los datos sobre las preferencias relacionales de género en la elección en función de la naturaleza de las actividades.

Tabla I. Distribución de las preferencias relacionales de elección del alumnado

\begin{tabular}{lcc}
\hline \multirow{2}{*}{ Actividades } & \multicolumn{2}{c}{$\begin{array}{c}\text { Preferencias Relacionales } \\
\text { de Elección \% }\end{array}$} \\
\cline { 2 - 3 } & Chicas & Chicos \\
\hline Tener una relación de pareja & 28.2 & 17.0 \\
Estudiar juntos & 73.3 & 61.6 \\
Compartir un secreto & 69.3 & 51.1 \\
Sentarse juntos en la clase & 77.0 & 70.2 \\
Prestarse la ropa & 34.7 & 22.0 \\
Ordenar y limpiar & 39.8 & 29.7 \\
Practicar deporte juntos & 57.3 & 66.6 \\
Jugar a un videojuego & 46.1 & 66.4 \\
Ser delegado/a de la clase & 49.1 & 43.1 \\
Ir de acampada & 60.3 & 52.0 \\
\hline
\end{tabular}

Los datos muestran que los porcentajes de elección de las chicas son superiores para todas las actividades excepto para jugar videojuegos y practicar deporte, lo que indica que son más preferidas en actividades puramente escolares como estudiar juntos, sentarse juntos o ser delegados de la clase, y en actividades de ámbito más privado, como son compartir secretos, prestarse ropa, tener una relación de pareja, ordenar y limpiar. Estos resultados revelan la presencia de patrones de género en la elección según la naturaleza relacional o social de las actividades.

En un análisis más pormenorizado, podemos conocer no sólo el género de las personas elegidas sino también de las electoras con el objeto de tener un mayor conocimiento de las preferencias mutuas del alumnado. Las figuras 1 y 2 presentan las elecciones de género que hacen las chicas y los chicos adolescentes según la naturaleza de la actividad. 


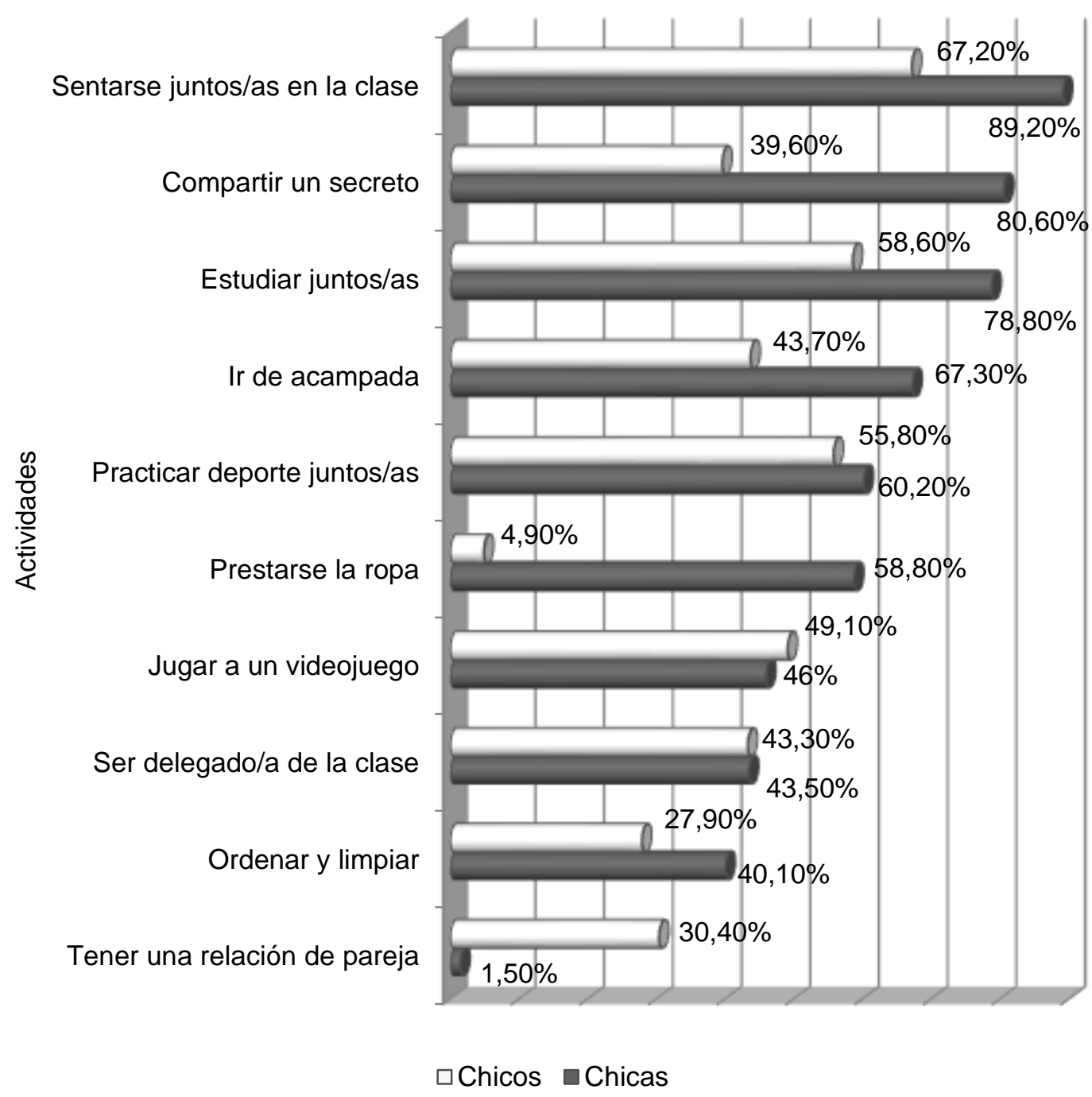

Figura 1. Preferencias relacionales de elección de las mujeres según tipo de actividad y género 


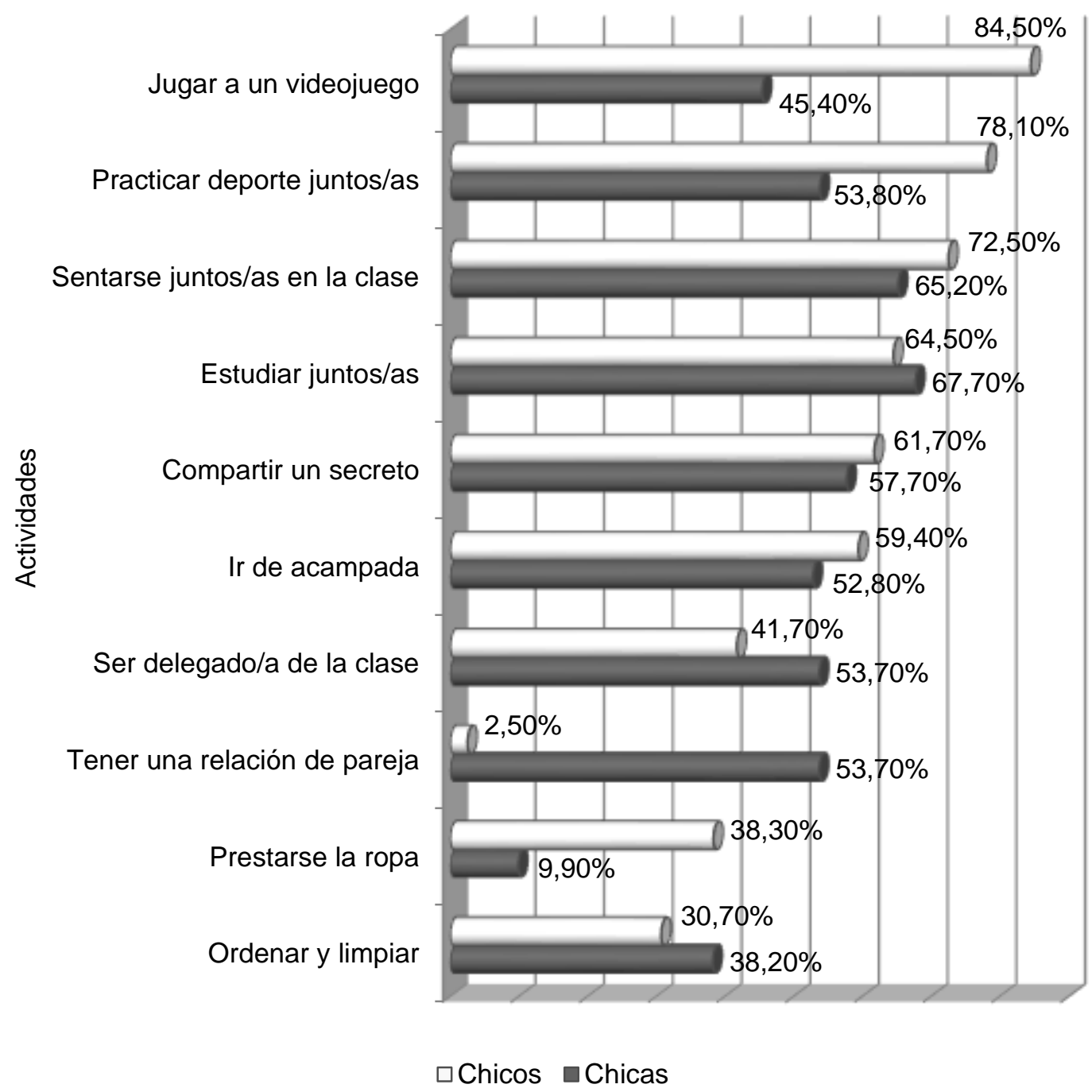

Figura 2. Preferencias relacionales de elección de los hombres según tipo de actividad y género

Se observa que los porcentajes de elección mutua entre adolescentes del mismo sexo son superiores a los porcentajes de elecciones de personas de distinto sexo, indicando una tendencia a mantener ámbitos de actividad separados. De este modo, las chicas se eligen mutuamente para sentarse juntas (89.2\%), compartir secretos (80.6\%), estudiar juntas (78.8\%) e ir de acampada (67.3\%); mientras que los chicos se eligen entre sí para jugar a videojuegos (84.5\%), practicar deporte juntos (78.1\%), sentarse juntos (72.5\%) y estudiar juntos (64.5\%). Esto indica que las chicas eligen principalmente a otras chicas para compartir secretos, a la vez que los chicos se eligen principalmente para jugar videojuegos y practicar deporte. Esta tendencia también se observa en las actividades definidas por el contexto escolar como sentarse juntos y estudiar juntos. La tabla II muestra que las diferencias observadas en las preferencias relacionales entre chicos y chicas son estadísticamente significativas. 
Tabla II. Tabla de contingencia según el género del alumnado ( $n=710)$ *

\begin{tabular}{|c|c|c|c|c|}
\hline $\begin{array}{l}\text { Preferencias Relacionales } \\
\text { de Elección }\end{array}$ & & $\begin{array}{l}\text { Mujeres } \\
(n=355)\end{array}$ & $\begin{array}{l}\text { Hombres } \\
(n=355)\end{array}$ & Significación \\
\hline Tener una relación de pareja & $\begin{array}{l}\text { Chicas } \\
\text { Chicos }\end{array}$ & $\begin{array}{l}5(1.5 \%) \\
99(30.4 \%)\end{array}$ & $\begin{array}{l}174(53.7 \%) \\
8(2.5 \%)\end{array}$ & $\begin{array}{l}X(2)=233.010 \\
P=.000 \\
C . \text { Contingencia }=.670\end{array}$ \\
\hline Prestarse la ropa & $\begin{array}{l}\text { Chicas } \\
\text { Chicos }\end{array}$ & $\begin{array}{l}191(58.8 \%) \\
16(4.9 \%)\end{array}$ & $\begin{array}{l}32(9.9 \%) \\
123(38.3 \%)\end{array}$ & $\begin{array}{l}X(2)=192.232 \\
p=.000 \\
\text { C. Contingencia }=.589\end{array}$ \\
\hline Compartir un secreto & $\begin{array}{l}\text { Chicas } \\
\text { Chicos }\end{array}$ & $\begin{array}{l}262(80.6 \%) \\
129(39.6 \%)\end{array}$ & $\begin{array}{l}187(57.7 \%) \\
200(61.7 \%)\end{array}$ & $\begin{array}{l}X(2)=27.830 \\
P=.000 \\
\text { C. Contingencia }=.186\end{array}$ \\
\hline Ir de acampada & $\begin{array}{l}\text { Chicas } \\
\text { Chicos }\end{array}$ & $\begin{array}{l}218(67.3 \%) \\
142(43.7 \%)\end{array}$ & $\begin{array}{l}172(52.8 \%) \\
192(59.4 \%)\end{array}$ & $\begin{array}{l}X(2)=12.889 \\
p=.000 \\
\text { C. Contingencia }=.132\end{array}$ \\
\hline Jugar un videojuego & $\begin{array}{l}\text { Chicas } \\
\text { Chicos }\end{array}$ & $\begin{array}{l}149(46 \%) \\
160(49.1 \%)\end{array}$ & $\begin{array}{l}148(45.4 \%) \\
272(84.5 \%)\end{array}$ & $\begin{array}{l}X(2)=12.427 \\
P=.000 \\
\text { C. Contingencia }=.129\end{array}$ \\
\hline Practicar deporte juntos & $\begin{array}{l}\text { Chicas } \\
\text { Chicos }\end{array}$ & $\begin{array}{l}195(60.2 \%) \\
182(55.8 \%)\end{array}$ & $\begin{array}{l}175(53.8 \%) \\
253(78.1 \%)\end{array}$ & $\begin{array}{l}X(2)=9.477 \\
P=.002 \\
\text { C. Contingencia }=.108\end{array}$ \\
\hline Sentarse juntos en la clase & $\begin{array}{l}\text { Chicas } \\
\text { Chicos }\end{array}$ & $\begin{array}{l}290(89.2 \%) \\
219(67.2 \%) \\
\end{array}$ & $\begin{array}{l}212(65.2 \%) \\
235(72.5 \%) \\
\end{array}$ & $\begin{array}{l}X(2)=8.699 \\
p=.003 \\
\text { C. Contingencia }=.095\end{array}$ \\
\hline
\end{tabular}

*12 estudiantes no especifican el género.

Como puede verse en la tabla III, las diferencias observadas en las elecciones se mantienen en el tramo de edad de 12 a 14 años (adolescencia temprana) tanto en actividades propias del contexto escolar como sentarse juntos/as en clase o practicar deporte juntos/as como en otras actividades sociales como tener una relación de pareja, prestarse ropa, compartir un secreto, ir de acampada o jugar a videojuegos; lo que parece indicar una escasa experiencia en relaciones amistosas con personas de distinto sexo entre adolescentes.

Tabla III. Tabla de contingencia según el género y la edad del alumnado: 12 a 14 años ( $n=456$ ***

\begin{tabular}{|c|c|c|c|c|}
\hline \multirow[b]{2}{*}{$\begin{array}{l}\text { Preferencias Relacionales } \\
\text { de Elección }\end{array}$} & \multicolumn{3}{|c|}{ 12-14 años } & \multirow[b]{2}{*}{ Significación } \\
\hline & & $\begin{array}{l}\text { Mujeres } \\
(n=230)\end{array}$ & $\begin{array}{l}\text { Hombres } \\
(n=226)\end{array}$ & \\
\hline Tener una relación de pareja & $\begin{array}{l}\text { Chicas } \\
\text { Chicos }\end{array}$ & $\begin{array}{l}5(2.2 \%) \\
76(33.6 \%)\end{array}$ & $\begin{array}{l}113(52.1 \%) \\
5(2.3 \%)\end{array}$ & $\begin{array}{l}X(2)=159.724 \\
p=.000 \\
\text { C. Contingencia }=.667\end{array}$ \\
\hline Prestarse la ropa & $\begin{array}{l}\text { Chicas } \\
\text { Chicos }\end{array}$ & $\begin{array}{l}131(58 \%) \\
12(5.3 \%)\end{array}$ & $\begin{array}{l}24(11.1 \%) \\
82(38.3 \%)\end{array}$ & $\begin{array}{l}X(2)=123.215 \\
P=.000 \\
\text { C. Contingencia }=.575\end{array}$ \\
\hline Compartir un secreto & $\begin{array}{l}\text { Chicas } \\
\text { Chicos }\end{array}$ & $\begin{array}{l}180(79.6 \%) \\
87(38.5 \%)\end{array}$ & $\begin{array}{l}122(56.2 \%) \\
139(64.1 \%)\end{array}$ & $\begin{array}{l}X(2)=23.038 \\
p=.000 \\
\text { C. Contingencia }=.204\end{array}$ \\
\hline Ir de acampada & $\begin{array}{l}\text { Chicas } \\
\text { Chicos }\end{array}$ & $\begin{array}{l}156(69 \%) \\
98(43.4 \%)\end{array}$ & $\begin{array}{l}112(51.1 \%) \\
134(62 \%)\end{array}$ & $\begin{array}{l}X(2)=12.685 \\
P=.000 \\
\text { C. Contingencia }=.157\end{array}$ \\
\hline Jugar a un videojuego & $\begin{array}{l}\text { Chicas } \\
\text { Chicos }\end{array}$ & $\begin{array}{l}120(53.1 \%) \\
122(54 \%)\end{array}$ & $\begin{array}{l}111(50.7 \%) \\
186(86.5 \%)\end{array}$ & $\begin{array}{l}X(2)=8.122 \\
p=.004 \\
\text { C. Contingencia }=.122\end{array}$ \\
\hline Practicar deporte juntos & $\begin{array}{l}\text { Chicas } \\
\text { Chicos }\end{array}$ & $\begin{array}{l}154(68.1 \%) \\
135(59.7 \%)\end{array}$ & $\begin{array}{l}125(57.3 \%) \\
169(77.9 \%)\end{array}$ & $\begin{array}{l}X(2)=6.775 \\
p=.009 \\
\text { C. Contingencia }=.107\end{array}$ \\
\hline Sentarse juntos en la clase & $\begin{array}{l}\text { Chicas } \\
\text { Chicos }\end{array}$ & $\begin{array}{l}197(87.2 \%) \\
148(65.5 \%)\end{array}$ & $\begin{array}{l}134(61.5 \%) \\
148(68.2 \%)\end{array}$ & $\begin{array}{l}X(2)=5.719 \\
p=.017\end{array}$ \\
\hline
\end{tabular}


**72 estudiantes no especifican la edad.

La tabla IV muestra que las diferencias de género observadas en las preferencias relacionales de adolescentes de 15 a 17 años son estadísticamente significativas para el conjunto de actividades antes señaladas, lo que indica que el establecimiento de relaciones amistosas y de pareja con personas del otro sexo en este período no parece influir en su tendencia a elegir a personas del mismo sexo con carácter general.

Tabla IV. Tabla de contingencia según el género y la edad del alumnado: 15 a 17 años ( $n=194)$ **

\begin{tabular}{|c|c|c|c|c|}
\hline \multirow{2}{*}{$\begin{array}{l}\text { Preferencias Relacionales } \\
\text { de Elección }\end{array}$} & \multicolumn{3}{|c|}{ 15-17 aก̃os } & \multirow[b]{2}{*}{ Significación } \\
\hline & & $\begin{array}{l}\text { Mujeres } \\
(n=96)\end{array}$ & $\begin{array}{l}\text { Hombres } \\
(n=98)\end{array}$ & \\
\hline Tener una relación de pareja & $\begin{array}{l}\text { Chicas } \\
\text { Chicos }\end{array}$ & $\begin{array}{l}0(0.0 \%) \\
22(22.9 \%)\end{array}$ & $\begin{array}{l}54(55.7 \%) \\
2(2.1 \%)\end{array}$ & $\begin{array}{l}X(2)=84.177 \\
p=.000 \\
\text { C. Contingencia }=.720\end{array}$ \\
\hline Prestarse la ropa & $\begin{array}{l}\text { Chicas } \\
\text { Chicos }\end{array}$ & $\begin{array}{l}57(59.4 \%) \\
4(4.2 \%)\end{array}$ & $\begin{array}{l}5(5.2 \%) \\
34(35.1 \%)\end{array}$ & $\begin{array}{l}X(2)=65.634 \\
p=.000 \\
\text { C. Contingencia }=.629\end{array}$ \\
\hline Compartir un secreto & $\begin{array}{l}\text { Chicas } \\
\text { Chicos }\end{array}$ & $\begin{array}{l}79(82.3 \%) \\
41(42.7 \%)\end{array}$ & $\begin{array}{l}56(57.7 \%) \\
51(52.6 \%)\end{array}$ & $\begin{array}{l}X(2)=4.275 \\
p=.039 \\
\text { C. Contingencia }=.136\end{array}$ \\
\hline Ir de acampada & $\begin{array}{l}\text { Chicas } \\
\text { Chicos }\end{array}$ & $\begin{array}{l}60(62.5 \%) \\
44(46.3 \%)\end{array}$ & $\begin{array}{l}51(52.6 \%) \\
49(50.5 \%)\end{array}$ & $\begin{array}{l}X(2)=0.920 \\
p=.337 \\
\text { C. Contingencia }=.067\end{array}$ \\
\hline Jugar a un videojuego & $\begin{array}{l}\text { Chicas } \\
\text { Chicos }\end{array}$ & $\begin{array}{l}27(28.1 \%) \\
38(39.6 \%)\end{array}$ & $\begin{array}{l}28(28.9 \%) \\
76(78.4 \%)\end{array}$ & $\begin{array}{l}X(2)=3.892 \\
p=.048 \\
\text { C. Contingencia }=.150\end{array}$ \\
\hline Practicar deporte juntos & $\begin{array}{l}\text { Chicas } \\
\text { Chicos }\end{array}$ & $\begin{array}{l}41(42.7 \%) \\
47(49 \%)\end{array}$ & $\begin{array}{l}40(41.2 \%) \\
74(76.3 \%)\end{array}$ & $\begin{array}{l}X(2)=2.736 \\
p=.098 \\
\text { C. Contingencia }=.116\end{array}$ \\
\hline Sentarse juntos en la clase & $\begin{array}{l}\text { Chicas } \\
\text { Chicos }\end{array}$ & $\begin{array}{l}90(93.8 \%) \\
70(72.9 \%)\end{array}$ & $\begin{array}{l}68(70.1 \%) \\
77(79.4 \%)\end{array}$ & $\begin{array}{l}X(2)=2.665 \\
p=.102 \\
\text { C. Contingencia }=.093\end{array}$ \\
\hline
\end{tabular}

**72 estudiantes no especifican la edad.

\section{Discusión y conclusiones}

El propósito general de este estudio era conocer la predisposición del alumnado adolescente a establecer relaciones entre iguales según la naturaleza de la actividad y analizar la posible influencia de expectativas sociales de género en sus elecciones. Estudios previos (Bascón, Arias y De la Mata, 2013; Garaigordobil, 2012; Feiring, 1999; Navarro-Pertusa, 2004) han demostrado la importancia de las relaciones amistosas que se establecen en la adolescencia para el desarrollo de capacidades y destrezas para las relaciones de pareja, al ser un período clave para el establecimiento y consolidación de relaciones sociales con personas de distinto sexo.

Los resultados muestran que las chicas son más elegidas para sentarse juntos en clase, estudiar juntos y compartir un secreto; mientras que los chicos lo son para practicar deporte juntos, jugar a un videojuego y sentarse juntos en la clase. Esto muestra que las actividades para las que se prefiere a un chico son principalmente de ocio y tiempo libre, mientras que las chicas son más elegidas para tareas escolares. Paralelamente, se encontró que algunas de las actividades para las que son más elegidas las chicas tienen un fuerte componente de confianza y respeto mutuo, como compartir un secreto. Estos resultados concuerdan con estudios previos (Muñoz-Tinoco et al., 2008; Ruiz-Pinto et al., 2013) que muestran que las chicas destacan en sociabilidad (ayudar, 
preocuparse y promover diálogos y acuerdos) mientras que los chicos lo hacen en agresividad física y relacional; indicando un mayor protagonismo de las chicas en las actividades basadas en el cuidado y dedicación, y de los chicos en aquellas basadas en la competición.

Otros estudios (Garaigordobil, 2012; Inglés et al., 2008) demuestran que las chicas emplean más formas cooperativas en la resolución de conflictos entre iguales, mientras que los chicos tienden a resolver los conflictos de forma agresiva. Algunas investigaciones (Bascón, Saavedra y Arias, 2013; García-Bacete et al., 2010; Monjas et al., 2008) apuntan incluso la influencia de los estereotipos de género como dimensión clave para explicar el establecimiento de relaciones y las formas de gestión de los conflictos durante la adolescencia. Consideramos que sería de interés incluir en estudios posteriores medidas sobre deseabilidad social (Ferrando y Chico, 2000; Ferrer, Bosch, Ramis, Torres y Navarro, 2006) para determinar la posible influencia de esta variable en las relaciones amistosas que establecen los adolescentes con personas de distinto sexo.

Encontramos también que se producen más elecciones mutuas entre adolescentes del mismo sexo que entre adolescentes de distinto sexo. Esto nos muestra que son las chicas las que se eligen entre sí para actividades basadas en el cuidado, mientras que son los chicos los que se eligen mutuamente para actividades basadas en la competición. A excepción de tener una relación de pareja, la única actividad en la que las chicas eligen más a chicos es para jugar videojuegos, mientras que los chicos eligen más a las chicas para que sean delegadas de clase y ordenar y limpiar. Estos resultados apuntan a lo que otras autoras han denominado socialización diferencial de género (Bosch et al., 2006). Simón (2010) señala que hombres y mujeres aprendemos socialmente las atribuciones de los roles de género, lo que hace que el rol de reproducción y cuidado lo asumamos como una función social de las mujeres y el rol de producción y mando como un ámbito asociado a los hombres. De la Peña et al. (2011) encuentra que estos roles están interiorizados por adolescentes andaluces, siendo más fuerte en los chicos que en las chicas.

García-Pérez et al. (2010) incluso señala que los chicos muestran peores actitudes en el ámbito relacional que las chicas. Algunas investigaciones previas (Feiring, 1999; Navarro-Pertusa, 2004) han documentado que las personas que refieren mayor número de amistades del sexo opuesto en su infancia y adolescencia disponen de mejores capacidades y destrezas para las relaciones de pareja, suponiendo esto un aprendizaje de habilidades relacionales. Los resultados de nuestro estudio sobre la escasa presencia de elecciones cruzadas en las respuestas del alumnado adolescente encuestado sugieren que chicos y chicas mantienen ámbitos de actividad diferenciados con escasas situaciones de interacción.

En nuestro estudio se observa que las diferencias entre chicos y chicas se mantienen a lo largo de la adolescencia, no detectándose una evolución en las preferencias relacionales manifestadas por los estudiantes desde la adolescencia temprana a la tardía. En contraste con estos resultados, De la Peña et al. (2011) encuentran un efecto evolutivo en grupos de mayor edad como consecuencia del establecimiento de relaciones amistosas con personas de distinto sexo que se produce durante la adolescencia. No obstante, esta discrepancia puede ser debido a la naturaleza de las relaciones que se exploran en uno y otro estudio, mientras De la Peña et al. (2011) se centran en detectar diferentes formas de violencia en las relaciones durante la adolescencia, nuestro trabajo se centra en analizar la actitud o predisposición a relacionarse con personas de distinto sexo en actividades propias de su edad, con independencia de las formas de interacción que supongan.

Los resultados de este estudio, junto con los de investigaciones previas, muestran la necesidad de continuar desarrollando en los centros educativos programas de coeducación durante la adolescencia, ya que se aprecia la fuerte influencia de creencias estereotipadas acerca del género en sus preferencias relacionales. El hecho de que haya pocas elecciones cruzadas indica que chicos y chicas mantienen ámbitos de actividad separados en el propio seno de la escuela. Esto confirma lo que Subirats y Tomé (2007) sostienen sobre el largo camino que aún queda por recorrer para dar el salto de una escuela mixta a una escuela coeducativa. Durante la 
adolescencia aumenta el número de interacciones sociales, estableciéndose relaciones de gran trascendencia, como son las amistosas y las de pareja; por lo que parece conveniente incorporar acciones educativas encaminadas a favorecer relaciones igualitarias.

Algunos trabajos recientes, entre ellos el de Rebollo-Catalán (2013) señalan la necesidad de innovar en educación desde una perspectiva de género para procurar un sistema educativo de calidad con equidad, incorporando un conjunto de saberes, prácticas y estrategias en la agenda educativa que promuevan la igualdad real entre hombres y mujeres. Simón (2010) insiste en la importancia de educar con enfoque de género -coeducación para la igualdad- en la escuela para evitar la reproducción del sexismo y promover la igualdad entre mujeres y hombres, siendo preciso trabajar tanto en el currículo formal (contenidos, materiales, metodología y evaluación) como en el currículo oculto (relaciones, lenguaje, uso de espacios, cargos de poder y decisión, roles, estereotipos, funciones, etc.). En este sentido, sería aconsejable llevar a cabo actuaciones en materia de coeducación durante la adolescencia que se centren en el establecimiento de relaciones igualitarias, sanas -libres de violencia- y no estereotipadas; así como en la enseñanza de la ética, del cuidado y la autonomía personal, la emocionalidad y la afectividad, la resolución de conflictos y la convivencia pacífica (Ferrer y Bosch, 2013; Sastre y Moreno, 2002; Simón, 2010; Subirats y Tomé, 2007).

\section{Referencias}

Barberá, E. (2006). Aportaciones de la psicología al estudio de las relaciones de género. En C. Rodríguez-Martínez (Comp.), Género y currículo: aportaciones del género al estudio y práctica del currículo (pp. 59-76). Madrid: Akal.

Bascón, M., Arias, S. y De la Mata, M. (2013). Contenidos y modos conversacionales en adolescentes: debatiendo sobre conflictos grupales y violencia de pareja. Infancia y Aprendizaje. Journal for the Study of Education and Development, 36(4), 489-500.

doi:10.1174/021037013808200302

Bascón, M., Saavedra, J. y Arias, S. (2013). Conflictos y violencia de género en la adolescencia: análisis de estrategias discursivas y recursos para la coeducación. Profesorado. Revista de currículum y formación del profesorado, 17(1), 289-307. Recuperado de http://www.ugr.es/ recfpro/rev171COL2.pdf

Benenson, J. F. y Christakos, A. (2003). The greater fragility of females' versus males' closest same-sex friendships. Child Development, 74(4), 1123-1129. doi:10.1111/1467-8624.00596

Bonino, L. (2000). Varones, género y salud mental: deconstruyendo la "normalidad" masculina. En M. Segarra y A. Carabí (Eds.). Nuevas Masculinidades (pp. 41-64). Barcelona: Icaria.

Bosch, E., Ferrer, V. A. y Alzamora, A. (2006). El laberinto patriarcal: reflexiones teórico-prácticas sobre la violencia contra las mujeres. Barcelona: Anthropos.

Bosch, E., Ferrer, V. A., Ferreiro, V. y Navarro, C. (2013). La violencia contra las mujeres: el amor como coartada. Barcelona: Anthropos.

Callirgos, J. C. (2003). Sobre héroes y batallas: los caminos de la identidad masculina. En C. Lomas (Ed.), ¿Todos los hombres son iguales?: identidades masculinas y cambios sociales (pp. 55-82). Barcelona: Paidós Contextos.

Chaves, C. (2002). Autoridad y mediación femenina como práctica de la paz. Duoda: Revista d'estudis feministes, 23, 65-81. 
Coria, C. (2005). Otra vida es posible en la edad media de la vida. En C. Coria, A. Freixas y S. Covas. Los cambios en la vida de las mujeres: temores, mitos y estrategias (pp. 19-70). Buenos Aires: Paidós.

De la Peña, E. M., Ramos, E., Luzón, J. M. y Recio, P. (2011). Andalucía Detecta-Andalucía Previene: sexismo y violencia de género en la juventud. Sevilla: Instituto Andaluz de la Mujer.

De Lemus, S., Castillo, M., Moya, M., Padilla, J. L. y Ryan, E. (2008). Elaboración y validación del Inventario de Sexismo Ambivalente para adolescentes. International Journal of Clinical and Health Psychology, 8(2), 537-562. Recuperado de: http://www.aepc.es/ijchp/articulos pdf/ijchp-292.pdf

Feiring, C. (1999). Other-sex friendship networks and the development of romantic relationship in adolescence. Journal of Youth and Adolescence, 28(4), 495-512.

Ferrando, P. J. y Chico, E. (2000). Adaptación y análisis psicométrico de la escala de deseabilidad social de Marlowe y Crowne. Psicothema, 12(3), 383-389. Recuperado de www.psicothema.com/pdf/346.pdf

Ferrer, V. y Bosch, E. (2013). Del amor romántico a la violencia de género: para una coeducación emocional en la agenda educativa. Profesorado. Revista de currículum y formación del profesorado, 17(1), 105-122. Recuperado de http://www.ugr.es/ recfpro/rev171ART7.pdf

Ferrer, V. A., Bosch, E., Ramis, M. C., Torres, G. y Navarro, C. (2006). La violencia contra las mujeres en la pareja. Creencias y actitudes en estudiantes universitarios/as. Psicothema, 18(3), 359-366. Recuperado de www.psicothema.com/pdf/3223.pdf

Freixas, A. (2001). Entre el mandato y el deseo: la adquisición de la identidad sexual y de género. En C. Flecha y M. Núñez (Coords.), La educación de las mujeres: nuevas perspectivas (pp.23-31). Sevilla: Universidad de Sevilla.

Garaigordobil, M. (2012). Resolución de conflictos cooperativa durante la adolescencia: relaciones con variables cognitivo-conductuales y predictores. Infancia y Aprendizaje, 35(2), 151-165. doi:10.1174/021037012800217998.

Garaigordobil, M. y Aliri, J. (2013). Relaciones del sexismo con justificación de la violencia, y con otras formas de prejuicio como la dominancia social y el autoritarismo. Estudios de Psicología, 34(2), 127-139. doi:10.1174/021093913806751384

García-Bacete, F. J., Sureda, I. y Monjas, M. I. (2010). El rechazo entre iguales en la Educación Primaria: una panorámica general. Anales de psicología, 26(1), 123-136. Recuperado de http://www.um.es/analesps/v26/v26 1/15-26 $1 . p d f$

García-Pérez, R., Rebollo-Catalán, A., Buzón-García, O., González-Piñal, R., Barragán-Sánchez, R. y Ruiz-Pinto, E. (2010). Actitudes del alumnado hacia la igualdad de género. Revista de Investigación Educativa, 28(1), 217-232. Recuperado de http://revistas.um.es/rie/article/view/98951

Herrera, M. C., Expósito, F. y Moya, M. (2012). Negative reactions of men to the loss of power in gender relations: Lilith vs. Eve. The European Journal of Psychology Applied to Legal Context, 4(1), $17-42$.

Inglés, C. J., Martínez-Monteagudo, M. C., Delgado, B., Torregrosa, M. S., Redondo, J., Benavides, G., García-Fernández, J. M. y García-López, L. J. (2008). Prevalencia de la conducta agresiva, conducta prosocial y ansiedad social en una muestra de adolescentes españoles: un estudio comparativo. Infancia y Aprendizaje, 31(4), 449-461. doi:10.1174/021037008786140968

Instituto Nacional de Estadística (2013). Mujeres y hombres en España. Madrid: Instituto Nacional 
de Estadística. Recuperado de

http://www.ine.es/ss/Satellite?L=es ES\&c=INEPublicacion C\&cid=1259924822888\&p=12547351

10672\&pagename=ProductosYServicios\%2FPYSLayout\&param1=PYSDetalleGratuitas

Lagarde, M. (2000). Claves feministas para la autoestima de las mujeres. Madrid: Horas y Horas.

Leaper, C. y Brown, C. S. (2008). Perceived experiences with sexism among adolescent girls. Child Development, 79(3), 685-704. doi:10.1111/j.1467-8624.2008.01151.x

Lomas, C. (2007). ¿La escuela es un infierno?: violencia escolar y construcción cultural de la masculinidad. Revista de Educación, 342, 83-101. Recuperado de

http://www.revistaeducacion.mec.es/re342/re342 05.pdf

Lorente, M. (2009). Los nuevos hombres nuevos: cómo adaptarse a los tiempos de igualdad. Barcelona: Destino.

Mañeru, A. (2007). La práctica de la autoridad en las relaciones. Crítica, 943, 55-59.

Martínez-Benlloch, I., Bonilla-Campos, A., Gómez-Sánchez, L. y Bayot, A. (2008). Identidad de género y afectividad en la adolescencia: asimetrías relacionales y violencia simbólica. Anuario de Psicología, 39(1), 109-118. Recuperado de http://www.raco.cat/index.php/AnuarioPsicologia/article/view/99374/159766

Mendoza, R., Batista-Foguet, J. M. y Rubio, A. (2006). La cooperación de los adolescentes en las tareas domésticas: diferencias de género y características asociadas. Cultura y Educación, 18(3-4), 363-379. doi:10.1174/113564006779172966

Monjas, M. I., Sureda, I. y García-Bacete, F. J. (2008). ¿Por qué los niños y las niñas se aceptan y se rechazan? Cultura y Educación, 20(4), 479-492. doi:10.1174/113564008786542181

Muñoz-Tinoco, M. V., Jiménez-Lagares, I. y Moreno, M. C. (2008). Reputación conductual y género en la adolescencia. Anales de Psicología, 24(2), 334-340. Recuperado de http://revistas.um.es/analesps/article/view/42891/41211

Navarro-Pertusa, E. (2004). Género y relaciones personales íntimas. En E. Barberá-Heredia y I. Martínez-Benlloch (Coords.), Psicología y Género (pp. 171-192). Madrid: Pearson Educación.

Noakes, M. A. y Rinaldi, C. M. (2006). Age and gender differences in peer conflict. Journal of Youth and Adolescence, 35(6), 881-891. doi:10.1007/s10964-006-9088-8

Olavarría, J. (2004). Modelos de masculinidad y desigualdades de género. En C. Lomas (Ed.), Los chicos también lloran: identidades masculinas, igualdad entre los sexos y coeducación (pp. 45-63). Barcelona: Paidós.

Oliva, A., Hidalgo, V., Martín, D., Parra, A., Ríos, M. y Vallejo, R. (2007). Ocio y tiempo libre: programa de apoyo a madres y padres de adolescentes. Sevilla: Consejería de Salud de la Junta de Andalucía. Recuperado de

http://www.juntadeandalucia.es/salud/sites/csalud/galerias/documentos/c 3 c 1 vida sana/ado lescencia/programa apoyo padres adolescentes/10 ocio.pdf

Oransky, M. y Marecek, J. (2009). "I'm not going to be a girl": masculinity and emotions in boys' friendships and peer groups. Journal of Adolescent Research, 24(2), 218-241.

doi:10.1177/074355840832995 
Parker, J. G., Low, C. M., Walker, A. R. y Gamm, B. K. (2005). Friendship jealousy in young adolescents: individual differences and links to sex, self-esteem, aggression, and social adjustment. Developmental Psychology, 41(1), 235-250. doi:10.1037/0012-1649.41.1.235

Perry, D. G. y Pauletti, R. E. (2011). Gender and Adolescent Development. Journal of Research on Adolescence, 21(1), 61-74. doi:10.1111/j.1532-7795.2010.00715.x

Rebollo-Catalán, A. (2006). Género e interculturalidad: educar para la igualdad. Madrid: La Muralla.

Rebollo-Catalán, A. (2013). La innovación educativa con perspectiva de género. Retos y Desafíos para el profesorado. Profesorado. Revista de Curriculum y Formación del Profesorado, 17(1), 3-8. Recuperado de http://www.ugr.es/ recfpro/rev171ed.pdf

Rodríguez-Mosquera, P. M. (2011). Códigos de honor masculinos y femeninos. Revista de Psicología Social, 26(1), 63-72. doi:10.1174/021347411794078499

Rose, A. J., Carlson, W. y Waller, E. M. (2007). Prospective association of co-rumination with friendship and emotional adjustment: considering the socioemotional trade-offs of corumination. Developmental Psychology, 43(4), 1019-1031. doi:10.1037/0012-1649.43.4.1019.

Ruiz-Pinto, E., García-Pérez, R. y Rebollo-Catalán, A. (2013). Relaciones de género de adolescentes en contextos escolares. Análisis de redes sociales con perspectiva de género. Profesorado. Revista de Curriculum y Formación del Profesorado, 17(1), 123-140. Recuperado de http://www.ugr.es/local/recfpro/rev171ART8.pdf

Sabuco, A., Sala, A., Santana, R. y Rebollo-Catalán, A. (2013). Discursos de niños varones sobre la masculinidad en contextos escolares. Un estudio piloto. Profesorado. Revista de Curriculum y Formación del Profesorado, 17(1), 141-157. Recuperado de http://www.ugr.es/ recfpro/rev171ART9.pdf

Salvador, T. (2009). Adolescentes y jóvenes: ocio y uso del tiempo libre en España. Recuperado de http://docplayer.es/180525-Adolescentes-y-jovenes-ocio-y-uso-del-tiempo-libre-en-espana.html

Sastre, G. y Moreno, M. (2002). Resolución de conflictos y aprendizaje emocional: una perspectiva de género. Barcelona: Gedisa.

Sau, V. (2000). De la facultad de ver al derecho de mirar. En M. Segarra y A. Carabí (Eds.), Nuevas masculinidades (pp. 29-40). Barcelona: Icaria.

Simón, M. E. (2008). Hijas de la igualdad, herederas de injusticias. Madrid: Narcea.

Simón, M. E. (2010). La igualdad también se aprende: cuestión de coeducación. Madrid: Narcea.

Soler, S. (2009). Los procesos de reproducción, resistencia y cambio de las relaciones tradicionales de género en Educación Física: el caso del fútbol. Cultura y Educación, 21(1), 31-42. doi:10.1174/113564009787531253

Subirats, M. y Tomé, A. (2007). Balones fuera; reconstruir los espacios desde la coeducación. Barcelona: Octaedro. 